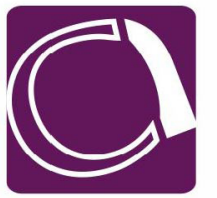

ENSAYO

\title{
FUERZA DE CARÁCTER Y CONTINUIDAD EMPRENDEDORA: EVIDENCIA DE ECUADOR
}

\section{GRIT AND ENTREPRENEURIAL CONTINUITY: EVIDENCE FROM} ECUADOR

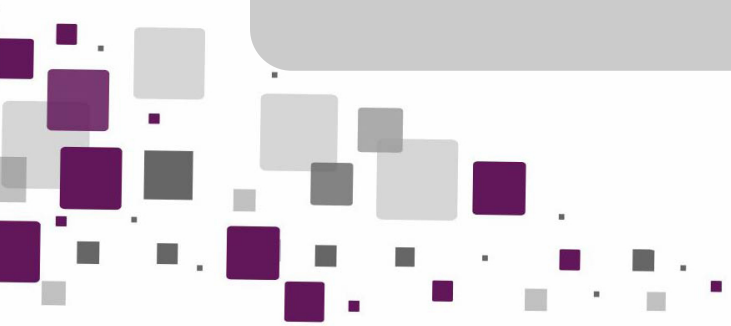

\section{Resumen / Abstract}

El objetivo del presente estudio es analizar si la fuerza de carácter, la tendencia de una persona a mostrar perseverancia en el esfuerzo, constancia en el interés y pasión en objetivos de largo plazo, influye en la continuidad emprendedora en Ecuador. Empleando una prueba chi-cuadrado, compara el nivel de fuerza de carácter de dos grupos de emprendedores, uno con iniciativas empresariales avanzadas y otro con iniciativas menos maduras, y demuestra que los emprendedores que tienen mayor fuerza de carácter, según evaluación de terceros, construyen empresas con mayor capacidad de seguir operando. Este hallazgo sugiere que, pese a competir en ecosistemas con limitaciones, personas con fuerza de carácter son capaces de superar obstáculos externos y consiguen que su iniciativa empresarial avance.

Palabras clave: continuidad emprendedora; emprendimiento; fuerza de carácter; resiliencia.

The purpose of this study is to analyse whether grit, the tendency of a person to show perseverance when making an effort, consistency when something is of interest, and passion when pursuing long-term goals influences entrepreneurial continuity in Ecuador. Using a chi-square test, the level of grit of two groups of entrepreneurs is compared, one with advanced business initiatives and the other one with less mature initiatives, showing that entrepreneurs with greater strength of character, according to third-party evaluations, build companies with greater capacity to continue operating. This finding suggests that, despite competing in ecosystems with limitations, people with grit can overcome external obstacles and make their entrepreneurship move forward.

Key words: entrepreneurial continuity; entrepreneurship; grit; resilience.

\section{- FABRIZIO NOBOA}

https://orcid.org/0000-0003-3298-1205 fnoboa@usfq.edu.ec

Business School. Universidad San Francisco de Quito. Ecuador. 


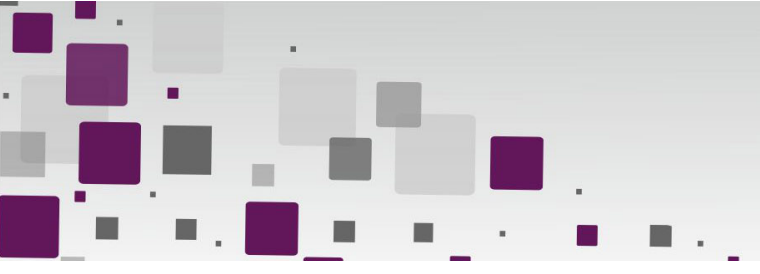

Introducción

El emprendimiento, es decir, la identificación, evaluación y explotación de oportunidades mediante nuevas formas de combinar recursos (Shane, 2012; Shane y Venkataraman, 2000), ha recibido mucha atención desde 1990 tanto en la esfera académica como en la práctica profesional. Concretamente, en Ecuador, como en muchos países del mundo, desde hace aproximadamente 15 años universidades e instituciones públicas y privadas han destinado tiempo y recursos para promoverlo entre sus ciudadanos. Se calcula que para el año 2018 el monto comprometido para emprendedores en el país ascendió a USD 41.05 millones, incluyendo capital semilla, inversión ángel y capital de riesgo (Alianza para el Emprendimiento y la Innovación, 2018). Sin embargo, pese a que el país presenta consistentemente una Tasa de Actividad Emprendedora Temprana (TEA) superior a sus pares de la región, la tasa de cierre de negocios también presenta niveles elevados, superiores al promedio de la región. Como consecuencia, pese a que cada vez se invierte más en emprendimiento y sus ideas, la mayoría muere en menos de 36 meses (Lasio et al., 2018).

No sólo las iniciativas emprendedoras en Ecuador tienen dificultad para permanecer en el tiempo. Tanto la teoría del capital humano como la ecología organizacional ofrecen algunas variables que explican la mortalidad de las iniciativas empresariales nuevas. Por un lado, la teoría del capital humano se centra en las características individuales del fundador como requisitos importantes para la supervivencia. Por otro, la ecología organizacional analiza el impacto de las características institucionales y las condiciones ambientales en la supervivencia de empresas recién creadas (Bruderl et al., 1992). Según ambas visiones, una iniciativa empresarial nueva será capaz de sobrevivir en función de las características individuales del fundador, las características organizacionales y las condiciones ambientales o del entorno.

En este sentido, el fenómeno de la "fragilidad de lo nuevo" (liability of newness en inglés) sostiene que el riesgo de morir de una organización es mayor al momento de su fundación y disminuye con su edad concretamente debido a la falta de experiencia del equipo fundador en la gestión de un negocio, la falta de confianza entre todos los miembros de la naciente organización
Año 10 | № 19 Enero - Junio 2022 | ISSN 2314 - 3738

Fabrizio Noboa y la falta de un portafolio estable de clientes (Stinchcombe, 1965). Estas debilidades explican la mayor tasa de mortalidad en empresas nuevas que en organizaciones maduras (Freeman et al., 1983).

De forma complementaria, según Shepherd et al. (2000), la tasa de mortalidad de una empresa nueva incrementa con el grado de novedad que la iniciativa emprendedora presenta en tres dimensiones específicas: novedad en el mercado, en la tecnología y en la gestión. La novedad en el mercado se refiere al grado de desconocimiento de los clientes sobre la nueva empresa; la novedad en la producción se refiere al nivel de experiencia y conocimiento de los miembros de la nueva empresa hacia la tecnología utilizada en la industria en la que compite; finalmente, la novedad en la gestión se refiere a la falta de habilidades de negocios, información específica de la industria y experiencia en sacar adelante una iniciativa emprendedora.

Másallá de las limitaciones organizacionales con que arranca una iniciativa emprendedora, la literatura también ha identificado condiciones ambientales que explican el cierre de algunas empresas nuevas. En Ecuador, por ejemplo, los dos factores externos más importantes que explican la mortalidad de las iniciativas emprendedoras son la falta de apoyo financiero y la baja rentabilidad del negocio, debido a altos niveles de competencia (Lasio et al., 2018). La situación económica del país posiblemente también incide, puesto que se ha encontrado que iniciativas emprendedoras que son lanzadas en economías con condiciones desfavorables tienen una mayor probabilidad de fracasar (Huggins et al., 2017). De forma semejante, entornos que presentan altos niveles de conflictividad política y violencia civil reducen la probabilidad de supervivencia de nuevas empresas (Hiatt y Sine, 2013).

Desde esta perspectiva, se podría hacer una lectura negativa al observar la existencia de múltiples factores internos y externos que dificultan la supervivencia y permanencia en el tiempo de una iniciativa emprendedora. Afortunadamente, los emprendedores pueden reaccionar, adaptarse a las demandas de un entorno cambiante y buscar cerrar la brecha entre sus recursos organizacionales iniciales y los exigidos para sobrevivir. Esta capacidad del emprendimiento para continuar operando recibe el nombre de continuidad emprendedora (Davidsson, 1991) y es el objetivo central del presente documento.

En particular, se ha encontrado que 
emprendedores que son capaces de crecer frente a la adversidad son quienes habitualmente mantienen su iniciativa a lo largo del tiempo (Bullough et al., 2014). Hayward et al. (2010) Ilaman resiliencia a la capacidad que permite a los emprendedores sobrellevar tiempos difíciles, sean recuperarse de un fracaso previo, persistir en presencia de múltiples obstáculos o solventar desafíos de gran envergadura. Sin embargo, la investigación que se encuentra en la intersección entre resiliencia y emprendimiento está muy fragmentada y dispersa en discusiones que definen ambos conceptos de formas muy diferentes. Por ello, se ha sugerido que, para avanzar en el conocimiento sobre emprendimiento y resiliencia, se empleen variables más fácilmente observables y que se vinculen con el aspecto particular de emprendimiento que se desea estudiar (Korber y McNaughton, 2018).

En esta línea, el campo de la psicología aporta con un concepto específico para medir el comportamiento vinculado con la resiliencia; dicho concepto se conoce como fuerza de carácter: la tendencia de una persona a mostrar perseverancia en el esfuerzo, constancia en el interés y pasión en objetivos de largo plazo (Duckworth et al., 2007).

Según esta visión, las personas que tienen fuerza de carácter tienden a ser más optimistas, más autocríticas con sus actuaciones y poseen una mentalidad que se centra en la perseverancia y en el continuo crecimiento personal. Como tal, emprendedores con mayor fuerza de carácter deberían ser capaces de sobrellevar obstáculos, haciendo que su iniciativa perdure en el tiempo.

Por las razones expuestas, el objetivo del presente estudio es analizar si la fuerza de carácter influye en la continuidad emprendedora en Ecuador, como motor que impulsa a los emprendedores a comportarse de una manera que multiplique la probabilidad de supervivencia de su iniciativa empresarial.

\section{Metodología}

Para verificar si la fuerza de carácter incide en la continuidad emprendedora, el presente estudio buscó emprendedores con iniciativas empresariales en marcha, que hayan comenzado operaciones aproximadamente en la misma fecha, pero que presenten distintos niveles de madurez. En general, emprendimientos con mayor nivel de madurez demuestran mayor capacidad para continuar operando (Davidsson, 1991).

La información provino gracias a una alianza de la universidad del autor del presente documento y una organización ecuatoriana sin fines de lucro que realiza intervención social a través de programas de educación, desarrollo territorial y emprendimiento. Uno de los programas de esta organización busca fortalecer y desarrollar competencias, habilidades y destrezas en emprendedores que desean, a través de sus negocios, generar riqueza para el país y mejorar su calidad de vida. En esencia, este programa se asienta en un proceso que incluye etapas de selección, capacitación y acompañamiento a cada idea de negocio, con el objetivo fundamental de apoyar su consolidación. El presente estudio recoge información del grupo de emprendedores que formaban parte del proceso de dicho programa en el periodo 2014-2017.

En diciembre del 2017, 72 emprendedores habían superado la etapa de convocatoria y capacitación, y se encontraban en la etapa de acompañamiento, mientras que 13 emprendedores habían decidido no seguir siendo parte del proceso de apoyo que brindaba la organización. Si bien los 72 emprendimientos iniciaron en el mismo punto de partida -presentación de la idea de negocio-, tres años más tarde algunos habían podido avanzar más rápidamente en su implementación al contar, por ejemplo, con un portafolio de clientes relativamente estable, procesos de producción medianamente estandarizados y bajo control, procesos de selección y capacitación de personal en marcha y contratos de largo plazo. Por diversos motivos, otros emprendimientos se habían rezagado, es decir, habían alcanzado un nivel menor de madurez en su iniciativa y apenas tenían ventas, enfrentaban problemas en la contratación, o no conseguían procesos fiables de producción, poniendo en duda su continuidad en el tiempo. Independientemente de la industria en la que competían, en el mismo lapso, algunos emprendedores habían conseguido construir empresas más maduras, con mayor capacidad de seguir operando en el futuro.

Con el fin de clasificar los emprendimientos en función de su capacidad de seguir operando, es decir, de su continuidad emprendedora, se solicitó a los 72 emprendedores que llenaran un informe de estatus de su iniciativa empresarial. Este informe, estándar en el proceso de acompañamiento de la organización sin fines de lucro, consultaba al emprendedor sobre el estatus de algunas áreas tradicionales de un negocio como marketing $y$ 
ventas, producción, recursos humanos y finanzas. El contacto y seguimiento del llenado del cuestionario se realizó por teléfono, desde la universidad y a nombre de la organización sin fines de lucro aliada, ofreciendo a los emprendedores dos entradas al cine para ver un estreno importante; estas entradas fueron gestionadas gracias a una alianza entre la universidad del autor del presente documento y una empresa local, y ofrecidas con el fin de mejorar la tasa de respuesta.

Tres meses más tarde, se recibieron 54 formularios, de los cuales 3 fueron eliminados porque estaban incompletos, dejando 51 para análisis, lo que equivale a una tasa final de respuesta del $70,8 \%$. Esta tasa podría parecer elevada; sin embargo, es preciso considerar que el bajo número de emprendedores en la muestra original exigía implementar mecanismos que elevaran la tasa de respuesta al máximo, con el fin de tener un número razonable de observaciones.

Posteriormente, se solicitó al Director de Área de la organización sin fines de lucro que clasificara a los emprendimientos en dos categorías, No Muy Avanzado y Avanzado, en función de la madurez de cada negocio según la información entregada en los formularios y su valoración cualitativa, al conocer a fondo la historia de todos los proyectos y el estatus deseado en el proceso de acompañamiento. Su conocimiento minucioso le otorgaba el poder de realizar esta clasificación de forma objetiva en su papel de experto en el área de interés. Su clasificación dejó 29 emprendedores en la categoría "No Muy Avanzados" y 22 en la categoría "Avanzados". Si bien ambos enfrentaban desafíos de todo tipo, el estatus de los "No Muy Avanzados" hacía pensar que probablemente no continuarían operando en el mediano plazo pese al apoyo y supervisión de la organización sin fines de lucro. En cambio, las expectativas de continuidad emprendedora de los "Avanzados" eran muy alentadoras.

Finalmente, se solicitó a dos mentores de cada emprendedor, quienes desconocían la clasificación realizada por el Director de Área pero que también conocían a fondo a cada emprendedor, que evaluaran la fuerza de carácter de sus pupilos empleando la escala de Angela Duckworth (Duckworth et al., 2007). Los dos mentores evaluaron a los 51 emprendedores usando la escala en español de 8-items. Cabe resaltar que la consistencia interna de la escala traducida es comparable a la original y válida para medir la tendencia de las personas a mantener el interés y el esfuerzo en objetivos de largo plazo (Arco-Tirado et al., 2018). Al medir la fuerza de carácter de los emprendedores mediante la valoración de terceras personas que los conocen bien, se evita caer en el sesgo de exceso de confianza que suelen presentar los emprendedores cuando realizan una evaluación de sus propias habilidades (Koellinger et al., 2007). De esta forma, se soslaya en un error habitual en estudios de emprendimiento.

Con el fin de evaluar si existe una relación entre el nivel de continuidad emprendedora (No Muy Avanzado / Avanzado) y la firmeza de carácter, se realizó una prueba chi-cuadrado entre el promedio de fuerza de carácter de cada categoría. Los resultados constan en la Tabla 1.

Tabla 1

Fuerza de carácter de los emprendedores, evaluación de terceras personas

\begin{tabular}{|l|l|l|}
\hline \multicolumn{2}{|l|}{ No Muy Avanzados } & Avanzados \\
\hline Media & 2.94 & 4.06 \\
\hline Varianza & 0.55 & 0.23 \\
\hline Observaciones & 29.00 & 22.00 \\
\hline Diferencia hipotética de las medias & 1.16 & \\
\hline Grados de libertad & 48.00 & \\
\hline Estadístico t & $-13,28$ & \\
\hline $\mathrm{P}(\mathrm{T}<=\mathrm{t})$ una cola & $5.4542 \mathrm{E}-18$ & \\
\hline Valor crítico de t (una cola) & 1.68 & \\
\hline $\mathrm{P}(\mathrm{T}<=\mathrm{t})$ dos colas & 0.00 & \\
\hline Valor crítico de t (dos colas) & 2.01 & \\
\hline
\end{tabular}

Nota. Elaboración del autor en base a resultados de Excel 
Los resultados de la prueba chi-cuadrado demuestran que el puntaje promedio que otorgan dos mentores en cuanto a la fuerza de carácter de sus pupilos difiere significativamente entre No Muy Avanzados y Avanzados (2.94 / 4.06). Esto demuestra que la fuerza de carácter tiene un impacto importante en la continuidad de una iniciativa emprendedora determinada: para continuar operando y alcanzar niveles de madurez más altos en su idea de negocio, los emprendedores deben mantener interés y esfuerzo para superar obstáculos internos y externos que dificultan la supervivencia de su iniciativa.

\section{Resultados y Discusión}

Toda persona que se encuentra frente a un obstáculo o que haya sufrido un revés siempre tiene dos opciones: abandonar o permanecer en el juego para enfrentar y superar la adversidad. Así como fundar una empresa se considera más emprendedor que no hacerlo, buscar el desarrollo continuo de ella es la opción más emprendedora cuando abstenerse de hacerlo es otra alternativa válida (Davidsson, 1991). Los resultados de este estudio demuestran que los emprendedores tienen diferentes niveles de fuerza de carácter, impactando en el grado de desarrollo de su iniciativa empresarial.

Desde esta óptica, es más probable que emprendedores con mayor fuerza de carácter sean capaces de superar los desafíos y obstáculos asociados al lanzamiento al mercado de una idea de negocio. Siguiendo la línea argumentativa que ofrece la literatura respecto a los factores que afectan la supervivencia de las empresas, el emprendedor debe superar tres categorías de desafíos: 1) sus características individuales como fundador; 2) las características de su organización; y 3) las condiciones ambientales o del entorno. A continuación, se presentan las implicaciones que tiene el contar con una mayor fuerza de carácter para enfrentar estos desafíos.

\section{Características Individuales y Fuerza de Carácter}

La teoría del capital humano sostiene que la educación del fundador y su experiencia previa en la industria son los factores de mayor impacto en la posibilidad de supervivencia de una iniciativa de negocio (Bruderl et al., 1992). Esto quiere decir que, si el emprendedor desea multiplicar las probabilidades de que su iniciativa empresarial sobreviva, debe elevar su nivel de formación y adquirir experiencia en la industria.

Sin embargo, si los motivos que llevaron al emprendedor a tener su propio negocio son justamente la falta de experiencia por ausencia de trabajo, como sucede en Ecuador y en varios países del mundo, se podría caer en un círculo vicioso del que resulta difícil salir. Los resultados de este estudio indican que existen emprendedores que son capaces de superar estas limitaciones y llevar hacia delante a su negocio, mientras otros no son capaces quizás porque carecen de fuerza de carácter.

En particular, adquirir conocimiento toma tiempo y exige un esfuerzo de reflexión, análisis y síntesis que algunas personas prefieren evitar. Según un estudio de Aaron Hochanadel y Dora Finamore (2015), aprender a veces se da en entornos con falta de apoyo, lo que dificulta la perseverancia; sin embargo, persistir es posible. Aquellas personas que creen que la inteligencia es fija, normalmente hacen menos esfuerzo para tener éxito; en cambio, quienes perseveran cuando se enfrentan a desafíos y adversidades suelen terminar aprendiendo.

La posibilidad de que existan emprendedores que estén menos dispuestos que otros a poner esfuerzo en aprender cosas nuevas puede explicarse porque algunos están convencidos en sus habilidades para sacar adelante la iniciativa emprendedora. Se ha encontrado que muchos emprendedores sobreestiman su probabilidad de éxito (Koellinger et al., 2007) y olvidan que deben y pueden actualizar sus habilidades, conocimiento y experiencia conforme avanzan en la puesta en marcha de su negocio. Como Koellinger et al. (2007) sugieren, este comportamiento puede explicarse porque los emprendedores tienen la tendencia a considerar su situación como única, lo que la hace difícilmente comparable con estadísticas actuales y futuras disponibles para elaborar pronósticos más precisos de su probabilidad de éxito. De hecho, los emprendedores realizan pronósticos propios de supervivencia que no están bien calibrados, es decir, que son demasiado optimistas (Hyytinen et al., 2014).

El presente estudio también quiso corroborar esta teoría $y$, durante el proceso de llenado del informe de estatus, se solicitó a los emprendedores que evaluaran su propia fuerza de carácter empleando la escala en español de 
emprender, y realizan acciones deliberadas para superar obstáculos relacionados con el aprendizaje y el crecimiento personal, dando continuidad a su iniciativa emprendedora.

\section{Características Organizacionales y Fuerza de Carácter}

Como se ha mencionado anteriormente, los factores organizacionales más comunes que dificultan la supervivencia de una iniciativa emprendedora nueva son la falta de conocimiento en la gestión del negocio, la falta de confianza entre todos los miembros de la organización y la falta de un portafolio estable de clientes (Stinchcombe, 1965). Superar estos obstáculos exige interés, esfuerzo y pasión, manifestados en comportamientos bajo el control del emprendedor.

Por ejemplo, la falta de experiencia en la gestión de un negocio puede reducirse mediante la adquisición de conocimiento de expertos, ya que emprendedores que han conversado e interactuado con ellos disponen de mejores capacidades de comunicación, lo que beneficia la gestión interna y externa del negocio (Ulvenblad et al., 2013). Cerrar esta brecha exige perseverancia, constancia y esfuerzo, ya que cuadrar agendas con gente valiosa no es simple y es preciso mantener varias conversaciones con objetivos claros, para poder sistematizar más tarde su contenido y poder extrapolar el aprendizaje más allá de lo anecdótico.

De forma semejante, existen múltiples mecanismos para superar la falta de confianza entre los miembros de la organización; algunos de ellos, como el coaching en un entorno grupal, consiguen resultados más rápido al fomentar cambios duraderos en los subordinados, como una resolución constructiva de conflictos y un mayor compromiso (Kets de Vries, 2005). Finalmente, un portafolio de clientes estable normalmente está atado a un nivel de compromiso elevado de la fuerza de ventas (Verbeke et al., 2011).

Ejecutar las acciones mencionadas exige perseverancia y esfuerzo por parte del emprendedor para conseguir resultados en el largo plazo. Tal es el caso de identificar expertos en la industria y persistir hasta ser recibido por ellos para tener una conversación útil; querer comprender a los subordinados, ponerse en sus zapatos y mostrar empatía; comprometer a la fuerza de ventas y encontrar las palabras adecuadas para motivarles, usando el lenguaje corporal adecuado y mostrando paciencia sin perder la exigencia.

Normalmente, las acciones citadas tienen resultados en el largo plazo, lo que podría desincentivar a quienes no tienen la capacidad de retrasar la gratificación. Esto quiere decir que habrá personas que han decidido sacar adelante una iniciativa emprendedora sin considerar las dificultades reales que implicaría y que, tras experimentar los desafíos citados, carecen de la fuerza de carácter para seguir adelante, poniendo en riesgo la continuidad de su iniciativa y sumándose así a la estadística de emprendimientos que no han sobrevivido.

A una conclusión similar se llega respecto a las dimensiones de novedad en el mercado, tecnología y la gestión del negocio que inciden en la mortalidad de emprendimientos nuevos (Shepherd et al., 2000). Superar estas fragilidades exige implementar acciones con esfuerzo e interés, pese a que sus resultados se verán en el largo plazo. Por ello, puede resultar perjudicial el énfasis que algunos medios de comunicación otorgan a los emprendimientos que son capaces de crecer de forma exponencial en poco tiempo; posiblemente, de tanto escuchar el mensaje, el emprendedor aspira a ser el "próximo unicornio" y olvida que para llegar allá debe superar el hecho de que sus potenciales clientes aún no lo conocen y que, por ejemplo, la implementación de un plan comercial y de marketing requiere conocimiento, esfuerzo, pasión, interés y normalmente no da resultados en el corto plazo.

En definitiva, para dar continuidad a su iniciativa emprendedora, es necesario demostrar constancia en el interés, esfuerzo y pasión para conseguir resultados en el largo plazo, ya que esto permitirá resolver cualquier desafío organizacional derivado de falta de recursos iniciales de una iniciativa empresarial nueva.

\section{Características Ambientales y Fuerza de Carácter}

Una de las relaciones más estudiadas en la literatura sobre emprendimiento es la existente entre el contexto y el desempeño de las iniciativas emprendedoras. Se ha llegado a acuñar el concepto de "ecosistema emprendedor" para referirse a todos los actores y variables que interactúan para promover este espíritu en una región determinada 
(Spilling, 1996). El estudio que anualmente lleva a cabo el Global Entrepreneurship Monitor dedica un amplio espacio a analizar la forma en que las condiciones del entorno alientan el desarrollo de emprendimientos en cada país. En el caso concreto de Ecuador, consistentemente el país recibe un índice bajo en esta dimensión (Lasio et al., 2018).

Pese a que es evidente que los emprendedores no operan en un vacío (Gartner, 1985), dedicar mucha atención al rol de los factores externos podría desviar innecesariamente la atención del emprendedor hacia factores sobre los que tiene menos control. En concreto, puede transmitir el mensaje de que los emprendimientos exitosos tienen lugar únicamente en ecosistemas con condiciones favorables, inclinando al emprendedor a pensar que, si compite en entornos desfavorables, la responsabilidad de construir emprendimientos exitosos radica en otros actores del ecosistema, especialmente si estos deben resolver problemas estructurales. Por ejemplo, si en un país los emprendimientos no prosperan por falta de financiamiento, el emprendedor podría llegar a la simplificación de que son los bancos y el gobierno quienes deben buscar alternativas para facilitar el trabajo a los emprendedores.

De esta manera, enfatizar los problemas estructurales que tiene un entorno para promover las iniciativas emprendedoras distrae al emprendedor de todas aquellas acciones que él/ella puede implementar para superar dichos obstáculos. Por ejemplo, un financiamiento reducido puede ser resuelto con un mayor esfuerzo para convencer de la viabilidad del negocio a familiares y amigos. Puede intentar resolverse con búsqueda de financiamiento externo o con participación en iniciativas de crowdfunding. Únicamente los emprendedores que están dispuestos a pasar por estos procesos que toman tiempo y esfuerzo podrán mover hacia delante su iniciativa empresarial. Aquellos que carezcan de fuerza de carácter posiblemente elijan dar un paso al costado y dejar de batallar.

Análogamente, emprendedores que dedican más esfuerzo a establecer una buena relación con proveedores y a analizar a fondo los riesgos del ingreso de nuevos competidores en su mercado tienen mayores probabilidades de generar una iniciativa empresarial que continúe (Gartner et al., 1999). Lo mismo puede afirmarse de emprendedores que deliberadamente implementan mecanismos para mitigar la ignorancia de los consumidores sobre su iniciativa empresarial (como diseminar oportunamente la información de sus productos mediante demostraciones personales), o para reducir la ignorancia de su equipo sobre la tecnología y los procesos de producción mediante entrenamiento continuo al personal adecuado, o para reducir la ignorancia sobre la gestión del negocio mediante el establecimiento de redes de contacto con empresarios, emprendedores y expertos (Shepherd et al., 2000).

Todas estas acciones, que exigen un elevado nivel de fuerza de carácter, reflejan un sincero interés en desarrollar continuamente la iniciativa empresarial y hacerla sobrevivir. La fuerza de carácter explica en parte por qué algunos emprendedores consiguen superar la fragilidad de la novedad, mientras que aquellos que encuentran obstáculos o dificultades deciden caminar más lento o abandonar la iniciativa.

\section{Conclusiones}

El presente estudio demuestra que emprendedores con un mayor nivel de fuerza de carácter son capaces de ubicar a su iniciativa empresarial en un nivel más alto de continuidad emprendedora. Comparado con pares que empezaron en el mismo punto de partida, que recibieron los mismos recursos y apoyo por parte de una organización sin fines de lucro, en el mismo horizonte de tiempo, los emprendedores con mayor fuerza de carácter llevaron su iniciativa empresarial más lejos.

Este hallazgo permite identificar algunas recomendaciones para organizaciones que financian y ayudan emprendedores en Ecuador y países similares, para medios de comunicación, para universidades y centros de enseñanza en emprendimiento y para emprendedores en sí mismos.

En primer lugar, las organizaciones que financian y apoyan a emprendedores podrían elegir a quien apoyar basadas en el nivel de la fuerza de carácter del candidato evaluado por terceras personas; debido al sesgo de sobreoptimismo, no es recomendable pedir que los emprendedores hagan una autoevaluación. Estas terceras personas pueden ser identificadas como referencias, y contactadas directamente para llenar el cuestionario. Potencialmente, emprendedores con mayor fuerza de carácter llegarán más lejos con su emprendimiento, extrayendo más provecho de los escasos recursos disponibles en el ecosistema ecuatoriano.

En segundo lugar, los medios de comunicación podrían dejar de valorar el esfuerzo 
emprendedor por la rapidez con la que hacer crecer el negocio o con la que consigue financiamiento. Es preferible destacar los obstáculos reales que ha superado el emprendedor y encomiar la perseverancia mostrada en situaciones concretas, por muy pequeñas que sean.

En tercer lugar, las universidades y centros de enseñanza de emprendimiento podrían establecer mecanismos pedagógicos que fomenten la fuerza de carácter en sus alumnos. Por ejemplo, solicitar que vuelvan a intentar una actividad que les resulta particularmente compleja y no abandonarla al primer esfuerzo o buscar una solución estándar en internet. Ciertas personas son propensas a no defender su punto de vista, a pedir ayuda a un colega que tiene mayor facilidad que ellas en ciertas áreas de negocio o a pedir el punto de vista del profesor, en lugar de perseverar buscando la información o intentarlo varias veces por sí mismas. Acciones tan pequeñas como felicitar la perseverancia o enfatizar el largo plazo pueden ayudar a construir emprendedores con más fuerza de carácter.

Finalmente, los emprendedores pueden trabajar en su fuerza de carácter. Esto se consigue mediante la perseverancia deliberada y enfocada; es decir, no se trata de perseverar porque sí, sino de perseverar para superar aquellos obstáculos que impiden el avance del emprendimiento, que presentan dificultad y que, una vez superados, darían al negocio mayor probabilidad de continuar en el futuro.

El presente estudio no está exento de limitaciones. Por ejemplo, carece de información cualitativa específica sobre cada emprendedor y su negocio, ya que, por convenios de confidencialidad con los candidatos, la organización sin fines de lucro no la pudo compartir para esta investigación. La información cualitativa podría haber ayudado a validar acciones específicas que los emprendedores "No Muy Avanzados" no han implementado o diferencias concretas con sus colegas "Avanzados", ofreciendo explicaciones complementarias tanto a su continuidad emprendedora como a su nivel de fuerza de carácter. En el futuro, sería deseable realizar investigaciones de carácter longitudinal que permitan hacer una combinación de información cualitativa y cuantitativa.

Por otro lado, el estudio emplea únicamente emprendedores radicados en Ecuador. Si bien los resultados podrían aplicarse a otros ecosistemas similares, es preciso tener cautela sobre la naturaleza de los emprendedores y sus iniciativas de negocio para extrapolar los resultados aquí obtenidos a otros perfiles.

Por último, podrían elaborarse modelos conceptuales más completos, añadiendo variables que impacten en la continuidad emprendedora, como los citados en el presente documento, con el fin de determinar la importancia relativa de la fuerza de carácter en presencia de otras variables.

\section{Referencias Bibliográficas}

Alianza para el Emprendimiento y la Innovación. (2018). La industria de capital emprendedor en Ecuador: 2018-2019. https://issuu.com/ martinalmirati/docs/brochure mapeo de fondos 2018-19 1

Arco-Tirado, J. L., Fernández-Martín, F. D. y Hoyle, R. H. (2018). Development and Validation of a Spanish Version of the Grit-S Scale. Frontiers in Psychology, 9, 1-7. https://doi. org/10.3389/fpsyg.2018.00096

Bruderl, J., Preisendorfer, P. y Ziegler, R. (1992). Survival Chances of Newly Founded Business Organizations. American Sociological Review, 57(2), 227-242. https://doi. org/10.2307/2096207

Bullough, A., Renko, M. y Myatt, T. (2014). Danger Zone Entrepreneurs: The Importance of Resilience and Self-Efficacy for Entrepreneurial Intentions. Entrepreneurship Theory and Practice, 38(3), 473-499. https:// doi.org/10.1111/etap.12006

Davidsson, P. (1991). Continued entrepreneurship: Ability, need, and opportunity as determinants of small firm growth. Journal of Business Venturing, 6(6), 405-429. https:// doi.org/10.1016/0883-9026(91)90028-C

Duckworth, A. L., Peterson, C., Matthews, M. D. y Kelly, D. R. (2007). Grit: Perseverance and passion for long-term goals. Journal of Personality and Social Psychology, 92(6), 1087-1101. https://doi.org/10.1037/0022$\underline{3514.92 .6 .1087}$

Freeman, J., Carroll, G. R. y Hannan, M. T. (1983). The Liability of Newness: Age Dependence 


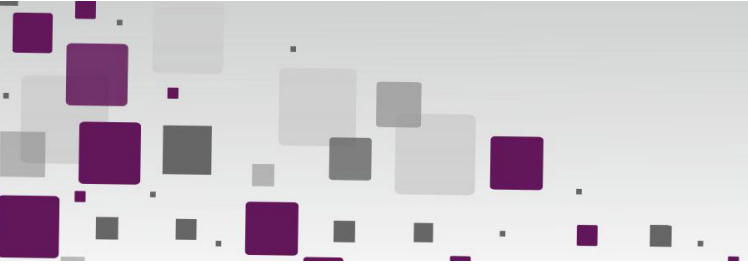

in Organizational Death Rates. American Sociological Review, 48(5), 692-710. https:// doi.org/10.2307/2094928

Gartner, W. B. (1985). A Conceptual Framework for Describing the Phenomenon of New Venture Creation. The Academy of Management Review, 10(4), 696-706. https://doi. org/10.2307/258039

Gartner, W. B., Starr, J. y Bhat, S. (1999). Predicting new venture survival: An analysis of "anatomy of a start-up" cases from Inc. Magazine. Journal of Business Venturing, 14(2), 215-232. https://doi.org/10.1016/ S0883-9026(97)00063-3

Hayward, M. L. A., Forster, W. R., Sarasvathy, S. D. y Fredrickson, B. L. (2010). Beyond hubris: How highly confident entrepreneurs rebound to venture again. Journal of Business Venturing, 25(6), 569-578. https://doi.org/10.1016/i. jbusvent.2009.03.002

Hiatt, S. R. y Sine, W. (2013). Clear and Present Danger: Planning and New Venture Survival amid Political and Civil Violence. Strategic Management Journal, 35(5), 773-785. https://doi.org/10.1002/smj.2113

Hochanadel, A. y Finamore, D. (2015). Fixed and Growth Mindset In Education And How Grit Helps Students Persist In The Face Of Adversity. Journal of International Education Research (JIER), 11(1), 47-50. https://doi. org/10.19030/iier.v11i1.9099

Huggins, R., Prokop, D. y Thompson, P. (2017) Entrepreneurship and the determinants of firm survival within regions: human capital, growth motivation and locational conditions. Entrepreneurship \& Regional Development, 29(3-4), 357-389. http://dx.doi.org/10.1080 L08985626.2016.1271830

Hyytinen, A., Lahtonen, J. y Pajarinen, M. (2014). Forecasting Errors of New Venture Survival. Strategic Entrepreneurship Journal, 8(4), 283-302. https://doi.org/10.1002/sej.1187

Kets de Vries, M. F. R. (2005). Leadership group coaching in action: The Zen of
Año 10 | N 19 Enero - Junio 2022 | ISSN 2314 - 3738

Fabrizio Noboa

creating high performance teams. Academy of Management Perspectives, 19(1), 61-76. https://doi.org/10.5465/ ame.2005.15841953

Koellinger, P., Minniti, M. y Schade, C. (2007). I think I can, I think I can: Overconfidence and entrepreneurial behavior. Journal of Economic Psychology, 28(4), 502-527. https://doi.org/10.1016/i.joep.2006.11.002

Korber, S. y McNaughton, R. B. (2018). Resilience and entrepreneurship: a systematic literature review. International Journal of Entrepreneurial Behavior \& Research, 24(7), 1129-1154. https://doi.org/10.1108/ iiebr-10-2016-0356

Lasio, V., Ordeñana, X., Caicedo, G., Samaniego, A. e Izquierdo, E. (2018). Global Entrepreneurship Monitor: Ecuador 2017. ESPAE-ESPOL. https://espae.espol.edu.ec/wp-content/ uploads/documentos/GemEcuador2017.pdf

Rauch, A. y Frese, M. (2000). Psychological approaches to entrepreneurial success. A general model and an overview of findings. En C. L. Cooper e I.T. Robertson (Eds.), International Review of Industrial and Organizational Psychology (pp. 101-142). Wiley.

Şahin, F., Karadağ, H. y Tuncer, B. (2019). Big five personality traits, entrepreneurial selfefficacy and entrepreneurial intention. International Journal of Entrepreneurial Behavior \& Research, 25(6), 1188-1211. https://doi.org/10.1108/ijebr-07-2018-0466

Shane, S. (2012). Reflections on the 2010 AMR Decade Award: Delivering on the Promise of Entrepreneurship as a Field of Research. The Academy of Management Review, 37(1), 1020. https://doi.org/10.5465/amr.2011.0078

Shane, S. y Venkataraman, S. (2000). The Promise of Entrepreneurship as a Field of Research. Academy of Management Review, 25(1), 217-226. https://doi. org/10.5465/amr.2000.2791611

Shepherd, D. A., Douglas, E. J. y Shanley, M. (2000). 
New venture survival: Ignorance, external shocks, and risk reduction strategies. Journal of Business Venturing, 15(5-6), 393-410. $\quad$ https://doi.org/10.1016/S0883$\underline{\text { 9026(98)00032-9 }}$

Spilling, O. R. (1996). The entrepreneurial system: On entrepreneurship in the context of a megaevent. Journal of Business Research, 36(1), 91-103. $\quad$ https://doi.org/10.1016/01482963(95)00166-2

Stinchcombe, A. (1965). Social structure and organizations. En J. G. March (Ed.), Handbook of organizations (pp. 142-193). RandMcNally.

Ulvenblad, P., Berggren, E. y Winborg, J. (2013). The role of entrepreneurship education and startup experience for handling communication and liability of newness. International Journal of Entrepreneurial Behavior \& Research, 19(2), 187-209. https://doi. org/10.1108/13552551311310374

Verbeke, W., Dietz, B. y Verwaal, E. (2011). Drivers of sales performance: a contemporary meta-analysis. Have salespeople become knowledge brokers? Journal of the Academy of Marketing Science, 39, 407-428. https:// doi.org/10.1007/s11747-010-0211-8 